\title{
Shoulder dystocia, a rare complication of ruptured uterus: a case report and review
}

\author{
Vani Aditya* \\ Department of Obstetrics and Gynecology, BRD Medical College, Gorakhpur, U.P., India
}

Received: 7 September 2013

Accepted: 21 September 2013

\author{
*Correspondence: \\ Dr. Vani Aditya \\ E-mail: drvaniaditya@gmail.com
}

(C) 2013 Aditya V. This is an open-access article distributed under the terms of the Creative Commons Attribution NonCommercial License, which permits unrestricted non-commercial use, distribution, and reproduction in any medium, provided the original work is properly cited.

\begin{abstract}
Shoulder dystocia is one of the most difficult complications of labor that is often unpredictable and hence unpreventable. In most cases, the cause is dystocia from the bony pelvis. The major brunt of complications is borne by the fetus. In neglected cases, grave maternal complications like obstructed labor and rupture of uterus may result. Very rarely, the reverse, uterine rupture leading to shoulder dystocia can also occur. The dystocia results from the soft tissue of the ruptured uterus itself. The present case is reported to emphasize the importance of early recognition of this condition. A gravida 3 para 2 woman presented in obstetrics emergency with delivery of head at home, six hours back, after being in labor for 8 to 10 hours. The uterine contractions ceased immediately following the delivery of the head. Her first delivery was by cesarean that was done for fetal distress. Second was an uneventful vaginal delivery at home. In this pregnancy, she presented with ruptured uterus .On laparotomy, the rupture was seen to involve the previous uterine scar. Lower segment was thick and previous uterine incision although transverse was situated higher up, above the lower segment. Rupture was repaired and patient recovered without any complications. In women with risk factors for uterine rupture, delivery should be conducted at hospitals with facilities for emergency cesarean. In this high risk group, if shoulder dystocia occurs, rupture of the uterus must be suspected as an underlying cause. Routine management protocols must be abandoned in favor of urgent laparotomy to improve the chances of fetal survival and save from litigation. At the same time, this case should not deter us from trial of labor after caesarean.
\end{abstract}

Keywords: Ruptured uterus, Shoulder dystocia, VBAC

\section{INTRODUCTION}

Shoulder dystocia is one of the most difficult complications of labor that is often unpredictable and hence unpreventable. In most cases, the cause is dystocia from the bony pelvis. The major brunt of complications is borne by the fetus. In neglected cases, grave maternal complications like obstructed labor and rupture of uterus may result. Very rarely, the reverse can also occur; uterine rupture may lead to shoulder dystocia. The dystocia results from the soft tissue of the ruptured uterus itself. The present case is reported to emphasize the importance of early recognition of this condition.

\section{CASE REPORT}

A gravida 3 para 2 woman presented in obstetrics emergency with spontaneous delivery of head six hours back, at home, after being in labor for 8 to 10 hours. Just after the delivery of head, pains subsided and the trunk failed to deliver. Within 15 minutes, she was rushed to a nearby primary health centre where she was diagnosed as a case of ruptured uterus with intrauterine death. No further attempt was made to deliver the trunk and she was referred to our tertiary care centre. Her first delivery was by cesarean that was done for fetal distress. The cesarean was done at a private hospital by a general surgeon. Birth weight of the baby was 2.6 kilograms. Second was an 
uneventful vaginal delivery at home. On examination, pulse rate was 124/min and BP, 80/62 mm Hg. Fetal parts were palpable superficially. Head was seen lying outside the introitus, in left occipitoposterior position (Figure 1). Diagnosis of ruptured uterus was made and patient was taken up for laparotomy.

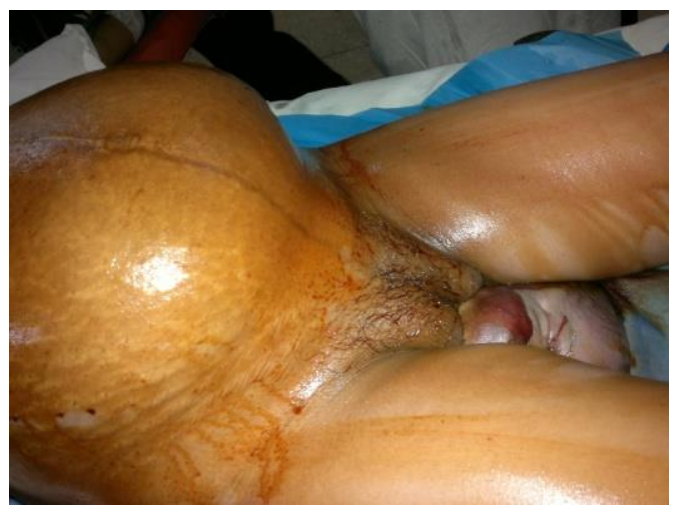

Figure 1: Shoulder dystocia in left occipitoposterior position in a woman with previous caesarean.

On entering the abdomen, the placenta was seen first and delivered. Next, the anterior shoulder overlying the trunk was seen with the back directed to the left. Breech was delivered followed by delivery of the trunk behind which the ruptured uterus was seen. Cleidotomy as well as vaginal delivery of the posterior arm could not be done because the cervix was firmly retracted over the neck. Meanwhile, an assistant attempted to replace the fetal head from below. The head was rotated, from left occipitoposterior to direct occipitoposterior position, flexed and pushed through the vagina into the lower segment but could not be pushed up further. Abdominal delivery was then completed by shoulder traction combined with jaw flexion. The baby was average in weight and the interscapular diameter was also normal. The rupture involved the previous uterine scar. The lower segment was thick and previous uterine incision although transverse was situated higher up, above the lower segment (Figure 2). Uterus was repaired and bilateral tubes ligated. Three units of PRBC were transfused. There was no intraoperative or postoperative complication. The patient was discharged on day 9 .

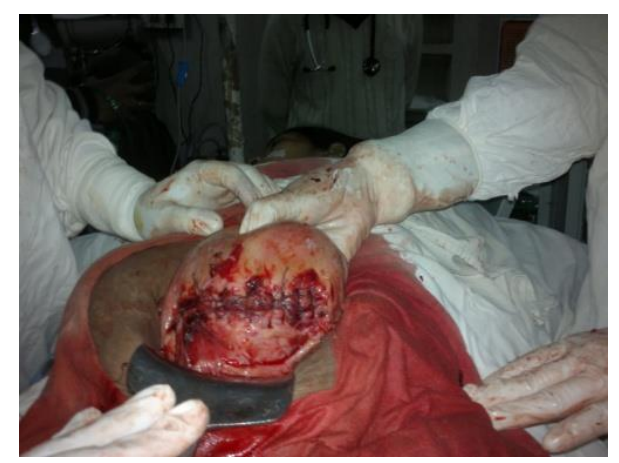

Figure 2: The transverse scar situated above the lower segment.

\section{DISCUSSION}

Shoulder dystocia is a rare obstetric complication that results when the anterior shoulder is arrested behind the pubic symphysis following delivery of the head. ${ }^{1}$ Very rarely, bilateral shoulder dystocia results when the descent of posterior shoulder is also checked by the sacral promontory from behind. Several antepartum risk factors like maternal diabetes, macrosomia and anencephaly as well as intrapartum risk factors like forceps delivery, prolonged duration of labor and precipitate labor have been associated with shoulder dystocia. Yet it may not be possible to predict shoulder dystocia in all the cases.

According to a report by the Confidential Enquiries into Stillbirths and Deaths in Infancy, $47 \%$ of deaths from shoulder dystocia were within five minutes of delivery of the head. ${ }^{2}$ Shoulder dystocia should therefore be promptly managed by protocol comprising of series of maneuvers that are used successively, based on the severity of shoulder dystocia. ${ }^{3}$ Among these, Zavanelli procedure is one of the last resort maneuvers. The method is principled on the reversal of delivery process by cephalic replacement i.e., return of fetal head to its previous intravaginal location. Delivery is then completed by the abdominal route. Success rate as high as $92 \%$ has been reported after cesarean ${ }^{4}$ In cases of failed cephalic replacement, abdominal rescue by hysterotomy to facilitate vaginal delivery was first described by O'Leary JA and Cuva A. ${ }^{5}$ Hysterotomy allows using more direct pressure to rotate the shoulders and assist them below the pubic symphysis followed by vaginal delivery of the baby. ${ }^{5,6}$ However, even this method may fail. In one such case, O'Shaughnessy M, managed by directly dislodging the anterior shoulder and hysterotomy facilitated vaginal delivery of posterior arm. ${ }^{7}$ Vollebergh $\mathbf{J}$ and van Dongen $\mathrm{P}$ failed in all attempts at abdominal rescue to vaginal delivery and finally managed abdominal delivery by extracting breech through the uterine incision and successful repeat cephalic replacement. $^{8}$

Rarely, a powerful uterine contraction may deliver the head but rupture the uterus. As a result, the fetal trunk and the shoulders are expelled outside the uterus and lie at right angles to the birth canal. This fails to deliver the shoulders and the trunk. Hence soft tissue of ruptured uterus and not the bony pelvis causes shoulder dystocia. Shoulder dystocia from ruptured uterus was first reported by Zisow DL. ${ }^{9}$ Rupture occurred in the unscarred uterus around the time of application of forceps that was followed by shoulder dystocia. Undiagnosed perforation during previous $\mathrm{D} \& \mathrm{C}$ was suspected to weaken uterine wall. However, there is a possibility that the uterine rupture was an unforeseen complication of forceps application as there were multiple successful vaginal deliveries even after the D\&C. The shoulders were present high up i.e., above the cervix and not behind the pubic symphysis which suggested rupture of the uterus and shoulder dystocia being the result. In another similar 
kind of case, a woman in her past two pregnancies, had successful VBAC that were uneventful and birth weights of the two babies were similar to the third which delivered following shoulder dystocia, i.e., $3.5 \mathrm{~kg}, 3.0 \mathrm{~kg}$ and $3.2 \mathrm{~kg}$. That the shoulder dystocia lead to uterine rupture therefore does not seem to be the case. In the presence of risk factor for uterine rupture i.e., history of previous cesarean, rupture of the uterus seems more likely to have caused shoulder dystocia. ${ }^{10}$

Though our patient did not deliver in presence of any obstetrician, the clinical details particularly suggest that rupture of the uterus was not the result but the cause of shoulder dystocia for the following reasons:

1. There were no features suggestive of obstructed labor before or following shoulder dystocia.

a. Labor was of normal duration in the first stage,

b. In the $2^{\text {nd }}$ stage, the uterine contractions ceased immediately following delivery of the head

c. Lower segment was thick and not enlarged or thinned out at laparotomy.

2. History suggestive of use of oxytocics, fundal pressure or any obstetric maneuvers to deliver the trunk was absent.

3. There was history of uneventful VBAC of average weight baby in the past.

4. Moreover, risk factors for uterine rupture were present but not for shoulder dystocia.

Whether shoulder dystocia or ruptured uterus, both need urgent management to salvage the fetus. While shoulder dystocia can be managed using series of maneuvers, ruptured uterus needs urgent laparotomy. In women with shoulder dystocia and risk factors for uterine rupture, ruptured uterus as the underlying cause must be suspected. Delivery of the fetus with shoulder dystocia may be more difficult in the presence of ruptured uterus as the fetus is expelled outside the uterine cavity and fails to align in maternal pelvis. Routine management protocols must be abandoned in favor of urgent laparotomy to improve the chances of fetal survival. Zavanelli procedure or abdominal rescue to vaginal delivery, one of the last resort maneuvers, should be the first in such a case. Importantly, very limited numbers of such cases have been reported and many of us may haven no previous experience regarding the delivery of fetus. Zisow DL managed to resolve shoulder dystocia in a case of ruptured uterus by abdominal rescue. One arm was manually directed into the uterus and then the birth canal. Vaginal delivery was then completed by Woods corkscrew maneuver. Mwenda AS resolved shoulder dystocia in a woman undergoing trial of labor after caesarean by delivering the posterior arm vaginally. ${ }^{10}$

Survival of the baby in ruptured uterus depends on the point at which the placenta separates and the decision delivery interval. As observed in a study, interval longer than 18 minutes resulted in demonstrable hypoxia and intervals longer than 30 minutes resulted in major neurological impairment. ${ }^{11}$ Regarding shoulder dystocia, studies have showed that 4 to 8 minutes is the critical time to resolve shoulder dystocia i.e., before the asphyxial brain damage occurs from compression of the carotid artery, cerebral hypoxia and death. ${ }^{1,12}$ In Mwenda's case, rupture was diagnosed after resolving shoulder dystocia but the fetal death could not be explained as the placenta was attached to the uterus even after the delivery. ${ }^{10}$ It is speculated that there was some delay in delivery when the attending midwives could not resolve shoulder dystocia and called the doctor or the fetus was dead at the time of presentation itself. Zisow DL performed laparotomy to resolve shoulder dystocia and delivered a live baby from the peritoneal cavity 31 minutes after the onset of bradycardia though the placenta had already separated. There was severe birth asphyxia with Apgar score of 1, 2 and 4. ${ }^{9}$

Though the imminent risk in uterine rupture and shoulder dystocia is to the fetus, the mother is also at risk of complications. Zisow DL's case was complicated with ureterouterine fistula that resulted from ischemic necrosis. ${ }^{9}$ However ischemia seems unlikely as the cesarean was done well enough in time to have even saved the baby. It is probable that the ureter got caught at cervical isthmus while repairing the torn vagina and cervix. In the other case, the patient died but the cause could not be ascertained.

In our case, the first delivery was by cesarean. Cesarean scar was transverse, not in the lower segment but a little higher up and thus inherently weak. The second delivery was at home and uneventful. This encouraged her to again go for home vaginal delivery in her third pregnancy which met with rupture uterus and a catastrophic fetal outcome. Though there were no maternal complications, a timely surgery could have saved the child.

To conclude, in presence of risk factors for uterine rupture, delivery should be conducted at hospitals with facilities for emergency cesarean. Rarely, when such cases are complicated with shoulder dystocia, uterine rupture as the underlying cause must be recognized early to improve the chances of fetal survival and save from litigation. At the same time, this case should not deter us from trial of labor after caesarean .A proper documentation and availability of the records would definitely be a help in selecting women for TOLAC. Besides, even the general surgeons should be well versed in the technique of lower segment caesarean.

\section{REFERENCES}

1. Dildy, Gary. Shoulder Dystocia. In: GDV Hankins, SL Clark, FG Cunningham, LC Gilstrap. Operative Obstetrics. Norwich, CT: Appleton \& Lange; 1995:233-56.

2. Confidential Enquiries into Stillbirths and Deaths in Infancy. Fifth Annual Report. London: Maternal and Child Health Research Consortium, 1998. 
3. O'Leary JA. Delivery techniques In: James A. O'Leary, editor. Shoulder Dystocia and Birth Injury: Prevention and Treatment. $3^{\text {rd }}$ ed. Florida: Humana Press; 2009:93.

4. Sandberg EC. The Zavanelli maneuver: 12 years of recorded experience. Obstet Gynecol. 1999;93:312317.

5. O'Leary JA and Cuva A. Abdominal rescue after failed cephalic replacement. Obstet Gynecol 1992; 80:514-6.

6. Enekwe A, Rothmund R, Uhl B. Abdominal Access for Shoulder Dystocia as a Last Resort - a Case Report. Geburtshilfe Frauenheilkd 2012;72:634-638

7. O'Shaughnessy M. Hysterotomy facilitation of the vaginal delivery of the posterior arm in a case of shoulder dystocia. Obstet Gynecol 1998; 92:693695.

8. Vollebergh $\mathrm{J}$ and van Dongen P. The Zavanelli maneuver in shoulder dystocia. Eur $\mathrm{J}$ Obstet Gynecol Reprod Biol.2000;89:81.

9. Zisow DL. Uterine rupture as a cause of shoulder dystocia. Obstet Gynecol.1996;87:818-819.
10. Mwenda AS. $4^{\text {th }}$ Stage Transvaginal omental herniation during VBAC complicated by shoulder dystocia: a unique presentation of uterine rupture BMC Pregnancy and Childbirth 2013;13:76.

11. Holmgren C, Scott JR, Porter TF, Esplin MS, Bardsley T. Uterine Rupture With Attempted Vaginal Birth After Cesarean Delivery: Decision-toDelivery Time and Neonatal Outcome. Obstet Gynecol. 2012 Apr;119(4):725-31.

12. Gurewitsch E, Allen R. Fetal manipulation for management of shoulder dystocia. Fetal Mat Med Rev 2006;17:239-280.

13. Leung TY, Stuart O, Suen SSH et al. Comparison of perinatal outcomes of shoulder dystocia alleviated by different type and sequence of manoeuvres: a retrospective review. $\mathrm{Br} \mathrm{J}$ Obstet Gynecol 2011;118:985-990.

DOI: $10.5455 / 2320-1770.1 j r \operatorname{cog} 20131242$

Cite this article as: Aditya V. Shoulder dystocia, a rare complication of ruptured uterus: a case report and review. Int J Reprod Contracept Obstet Gynecol 2013;2:691-4. 